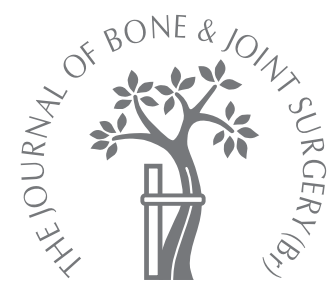

RESEARCH

\title{
Acetabular and proximal femoral alignment in patients with osteoarthritis of the dysplastic hip and its influence on the progression of disease
}
H. Aoki,
Y. Nagao,
S. Ishii,
T. Masuda,
M. Beppu

From St Marianna

University School of

Medicine, Kawasaki, Japan
H. Aoki, MD, PhD

Orthopaedic Surgeon, Professor (Emeritus)

- Y. Nagao, MD, PhD,

Orthopaedic Surgeon

S. Ishii, MD, PhD,

Orthopaedic Surgeon

T. Masuda, MD, PhD,

Orthopaedic Surgeon

M. Beppu, MD, PhD

Orthopaedic Surgeon,

Professor

St. Marianna University School of Medicine, 2-16-1, Sugao

Miyamae-ku, Kawasaki, 216-

8511, Japan.

Correspondence should be sent to Professor H. Aoki; e-mail:

h2aoki@marianna-u.ac.jp

(C)2010 British Editorial Society of Bone and Joint Surgery doi:10.1302/0301-620X.92B12 $23446 \$ 2.00$

$J$ Bone Joint Surg $[\mathrm{Br}]$ 2010;92-B:1703-9.

Received 17 September 2009;

Accepted after revision 8 July

2010

In order to evaluate the relationship between acetabular and proximal femoral alignment in the initiation and evolution of osteoarthritis of the dysplastic hip, the acetabular and femoral angles were calculated geometrically from radiographs of 62 patients with prearthrosis and early osteoarthritis. The sum of the lateral opening angle of the acetabulum and the neck-shaft angle was defined as the lateral instability index (LII), and the sum of the anterior opening angle of the acetabulum and the anteversion angle of the femoral neck as the anterior instability index (AII). These two indices were compared in dysplastic and unaffected hips. A total of 22 unilateral hips with pre-arthrosis were followed for at least 15 years to determine whether the two indices were associated with the progression of osteoarthritis.

The LII of the affected hips (197.4 (SD 6.0)) was significantly greater than that of the unaffected hips (1830 (SD 6.9)). A follow-up study of 22 hips with pre-arthrosis showed that only the LII was associated with progression of the disease, and an LII of 196 was the threshold value for this progression.

The anatomical relationship between the orientation of the acetabulum and the proximal femur in dysplastic hips is poorly understood, and its relationship to the progression of secondary osteoarthritis (OA) has not been studied. Previous studies have generally been confined to the estimation of the percentage cover of the femoral head by the acetabulum. The centre-edge angle, ${ }^{1}$ femoral head extrusion index, ${ }^{2}$ acetabular-head index, ${ }^{3}$ and anterior centre edge angle, ${ }^{4-6}$ are used and are smaller in dysplastic compared with normal hips. These indices give only a twodimensional indication of how the hip is being loaded; consequently, their influence on disease progression remains controversial..$^{7-13}$

Studies using CT have suggested that the anterior opening angle of the acetabulum is the same or nearly the same in osteoarthritic and normal hips, whereas the anteversion angle of the femoral neck is significantly greater in $\mathrm{OA}$ hips than normal hips. ${ }^{14-16}$ Three-dimensional (3D) evaluation of the morphology of the hip was introduced using modified inlet-view radiographs or 3D-reconstructed CT scans. These studies evaluated the angles of orientation of the acetabulum and acetabular cover of the femoral head qualitatively ${ }^{17,18}$ and quanti- tatively, ${ }^{19-21}$ but did not assess the relationship between the acetabular and the proximal femoral angles in each case. Of the studies using 3D-reconstructed CT, Abel et $\mathrm{al}^{20}$ measured the anterior opening angle of the acetabulum and both neck-shaft and neck anteversion angles of the proximal femur. However, neither the lateral opening angle of the acetabulum nor the relationship between these values was presented. Dandachli et al, ${ }^{21}$ on the other hand, calculated the anterior and lateral opening angles of the acetabulum and the percentage of acetabular cover of the femoral head, but did not fully evaluate the morphology of the proximal femur, nor did they analyse these values as predictors of the progression of OA. A study of the 3D anatomy of the dysplastic hip evaluated the acetabular diameter and 3D morphology of the proximal femur independently of the effects of total hip replacement, but not the relationship between the two elements. $^{22}$

So far there have been no studies that have fully assessed the relationship between acetabular orientation and the morphology of the femoral neck in the dysplastic hip. In this study we measured the anterior and lateral opening angles of the acetabulum using the 


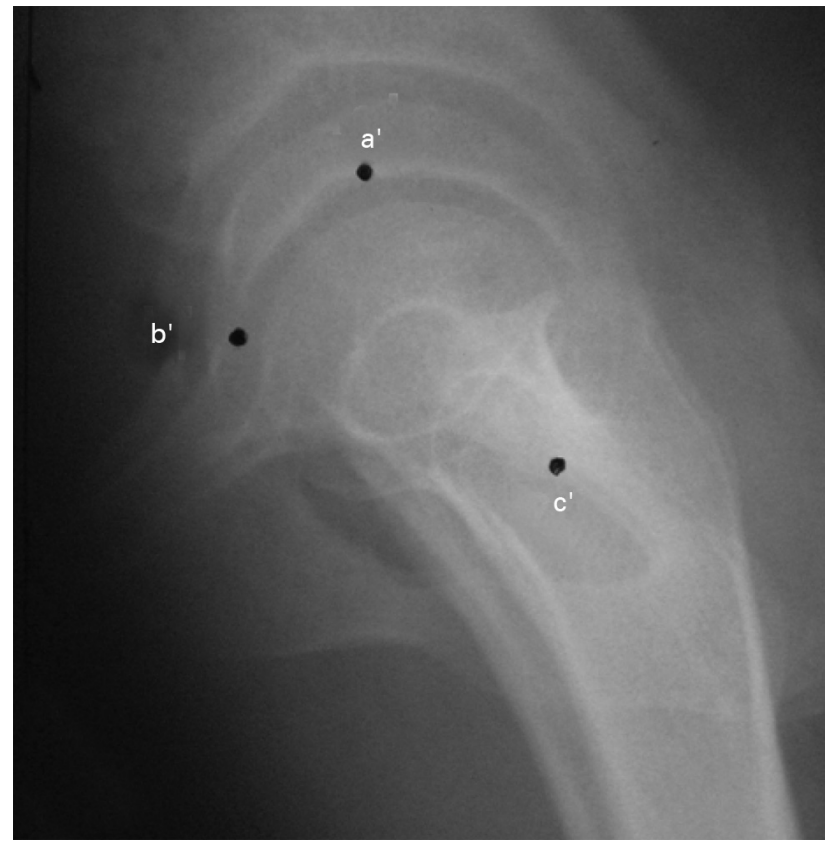

Fig. 1a

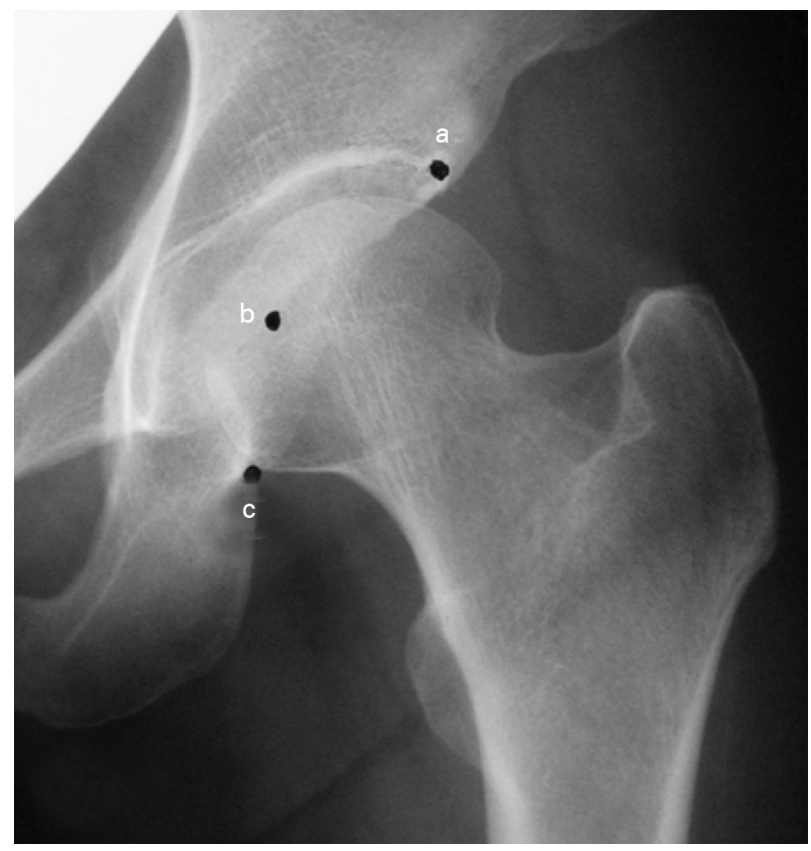

Fig. 1b

Lateral a) and posteroanterior (PA) b) radiographs of the hip. The most superior markers (a) and (a') are the highest points perpendicular to the anatomical reference plane (ARP) in both views. The anterior point (b') is determined next as the most anterior point of the rim of the acetabulum with respect to the ARP on the lateral radiograph. The point (b) is at the same level as ( $b^{\prime}$ ) on the anterior acetabular rim in the PA view. The inferior points (c) and $\left(c^{\prime}\right)$ are the lowest points of the posterior acetabular rim on the PA view, and a point at the same level in the posterior circular line of the acetabular rim in the lateral view. If bidirectional radiographs are taken accurately, these three points (a, b, c in PA and a', b', c' in lateral view) indicate the same anatomical bony landmarks in the true acetabulum.

method of Nagao et $\mathrm{al}^{23}$ in patients with early-stage OA. We evaluated the relationship between the angles of the acetabulum and the femoral neck using values of the anteversion angle of the femoral neck and the neck-shaft angle as described by $\mathrm{Kai}^{24}$ and Ogata and Goldsand. ${ }^{25}$

The study had two purposes: first, to evaluate the relationship between the lateral and anterior opening angles of the acetabulum, the neck-shaft and neck anteversion angles of the proximal femur in dysplastic OA hips, and second, to examine their ability to predict the onset of OA in the dysplastic hip.

\section{Patients and Methods}

Relationship between acetabular inclination and proximal femoral angles. All new patients who presented with prearthrosis or early OA secondary to hip dysplasia between 1975 and 1990 were included in the study. There were 62 patients ( 57 women, five men) with a mean age of 38.3 years (14 to 64 ). A total of 84 hips (35 pre-arthrosis and 49 early OA) were involved. There were 22 patients with bilateral disease: of their 44 hips, ten were graded prearthrosis and 34 early OA. Of the 40 unilateral cases, 25 were graded pre-arthrosis and 15 early OA. These hips will be referred to here as 'OA hips'. The 40 contralateral asymptomatic hips in unilaterally affected patients comprised the control group, and will be referred to as 'unaffected hips'. The theoretical weakness of using these hips as controls in a series of patients referred for the investigation of hip dysplasia is appreciated and will be discussed later. All participants gave informed consent for radiological examination.

Because the acetabulum is not a true hemisphere and the acetabular rim forms slight curves, we chose the most superior, anterior and inferior points on the acetabular rim to calculate the opening angles of the acetabulum. The inclination of the vertical axis to this triangular plane was defined as the acetabular inclination. The standing pelvic position was used as the anatomical reference plane when evaluating the acetabular inclination angles, on which three points, the two (right and left) anterior superior iliac spines and the pubic symphysis, were defined as the vertical and frontal planes.

Both posteroanterior (PA) and lateral radiographs were taken. The PA radiograph was taken with the patient lying prone with all three pelvis points in contact with the film. The lateral radiograph was taken in a true lateral position with the affected hips on the film. The $\mathrm{x}$-ray beam was directed perpendicular to the sagittal plane through the centre of both acetabula to obtain the lateral images. The affected side was that with the smaller acetabular diameter and was used to identify the three points.

Figure 1 shows the lateral and PA radiographs. In order to ensure that the three points $(a, b, c)$ on the PA radiograph 


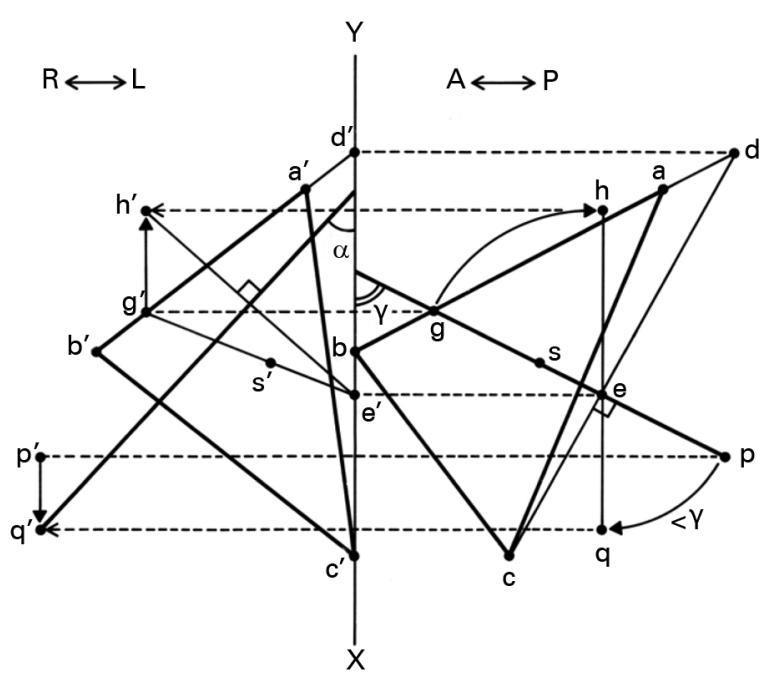

Fig. 2

Geometrical graphs for calculation from the two projection views. Triangles $(a, b, c)$ and $\left(a^{\prime}, b^{\prime}, c^{\prime}\right)$ are obtained from the radiographs. Line $\mathrm{Y}-\mathrm{X}$ indicates the reference line of axis in the posteroanterior (PA) view and the anatomical reference plane line in the lateral view. Point $(p)$ and $\left(p^{\prime}\right)$ in the PA and lateral views are projected points of the given point $(P)$, respectively. Angle $\alpha$ is the true anterior opening angle and $\gamma$ the projected lateral opening angle. The true lateral opening angle $\beta$ is calculated from $\cos \gamma \times \cos \alpha=\cos$ $\beta$, where the angle $\beta$ is obtained by using the trigonometric function $\cos \beta$. All points in both P-A and L-R plane are the projected points where two lines cross or a line intersect the triangular plane which was defined by three points on the acetabular rim.

corresponded to those $\left(a^{\prime}, b^{\prime}, c^{\prime}\right)$ on the lateral radiograph, it was essential to have clear images taken in the correct alignment. Using these points, the anterior and lateral opening angles were calculated geometrically using methods that we have previously reported (Fig. 2). ${ }^{23}$

The femoral anteversion and the true neck-shaft angles were calculated geometrically from PA and true lateral radiographs of the proximal femur using methods proposed by $\mathrm{Kai}^{24}$ and Ogata and Goldsand. ${ }^{25}$

In order to evaluate progression of the disease we used the four-stage radiological classification system described by Nozawa et $\mathrm{al}^{9}{ }^{9}$ where the strength of inter-observer agreement as described by the $\kappa$ statistic was reported as 'moderate'. ${ }^{26}$ In order to evaluate these relationships quantitatively we applied the instability index proposed by McKibbin, ${ }^{27}$ which he originally developed to evaluate instability leading to hip dislocation in neonates. This index is the sum of the anterior opening angle of the acetabulum and the anteversion angle of the femoral neck, with larger values indicating greater instability. In our study the sum of the anterior opening angle of the acetabulum and the anteversion angle was referred to as the anterior instability index (AII). The lateral instability index (LII), was the sum of the lateral opening and the neck-shaft angle. The two indices together indicate the degree of instability in the hip joint.

These indices were then compared between hips with OA and unaffected hips.
In order to estimate the reliability of this method of measuring the acetabular inclination angle, 40 randomly selected radiographs were assessed for inter- and intraobserver agreement of the anterior and lateral opening angles. Two authors (HA, YN) measured the same radiographs separately to determine inter-observer agreement, and one author (HA) measured the same radiographs twice for intra-observer agreement. We considered the method would be reliable if inter- and intra-observer agreement was high.

Influence of the relationship between the acetabular inclination and proximal femoral angles in progression to $O A$. In order to establish whether the instability indices are associated with progression to OA, all affected pre-arthrosis hips were followed prospectively. There were 35 such hips, of which ten were bilateral and were excluded to avoid the affected contralateral hip influencing the progression of pre-arthrosis. This left 25 patients with unilateral prearthrosis initially, three of whom were lost to follow-up. The remaining 22 patients were all women, with a mean age of 35.5 years (22 to 50), who were followed for a mean of 194 months (181 to 223). We assessed six factors: age, LII, AII, Sharp angle, ${ }^{28}$ centre-edge angle and acetabular head index at initial examination, and looked for any correlation with progression from pre-arthrosis to OA.

In order to evaluate progression of the disease we used the four-stage radiological classification system described by Takatori et al, ${ }^{26}$ where the strength of the inter-observer agreement as described by the $\kappa$ statistic was reported as 'moderate'. Two authors (HA, YN) followed up all patients and evaluated the radiographs independently. OA was deemed to have progressed if both observers agreed.

Statistical analysis. Student's $t$-test was used to compare the indices of OA and unaffected hips. The intraclass correlation coefficient for inter- and intra-observer reliability was calculated using SPSS version 15.0J (SPSS Japan, Tokyo, Japan). In order to determine whether other factors were associated with progression to OA in the 22 hips, six factors: age, LII, AII, Sharp angle, centre-edge angle and acetabular head index were examined using binary logistic analysis (SPSS 15.0J). Statistical significance was assumed if $\mathrm{p}<0.05$. Nagelkerke $\mathrm{R}^{2}$ (coefficient of determination) ${ }^{29}$ was calculated to obtain a threshold value for univariate factors significantly associated with progression.

The Bioethics Committee of St Marianna University School of Medicine approved the use of patient data for this study.

\section{Results}

Comparison of All and LII in OA and unaffected hips. The inter-class correlation coefficients of both inter- and intraobserver reliability for the anterior opening and lateral opening angles of the acetabulum are shown in Table I. This measurement method was considered reliable.

The AII and LII proved to be independent variables, given that there was no clear correlation between them in 
Table I. Inter- and intra-observer agreement of the measurement of the acetabular inclination angles

\begin{tabular}{lll}
\hline & Inter-observer agreement & Intra-observer agreement \\
\cline { 2 - 3 } & ICC $^{*}\left(\mathbf{9 5} \% \mathbf{C I}^{\dagger}\right)$ & ICC $(\mathbf{9 5} \% \mathbf{C I})$ \\
\hline $\mathrm{LOA}^{\ddagger}$ & $0.936(0.883$ to 0.966$)$ & $0.942(0.893$ to 0.969$)$ \\
$\mathrm{AOA}^{\S}$ & $0.938(0.886$ to 0.967$)$ & $0.953(0.913$ to 0.975$)$ \\
\hline
\end{tabular}

* ICC, intraclass correlation coefficient

$+\mathrm{Cl}$, confidence interval

‡ LOA, lateral opening angle

$\S A O A$, anterior opening angle

Table II. Comparison of $\mathrm{LII}^{*}$ and $\mathrm{AlI}^{\dagger}$ between $\mathrm{OA}^{\ddagger}$ and unaffected hips

\begin{tabular}{lcccc}
\hline & OA hips & Unaffected hips & p-value \\
\hline Number of hips & 84 & 40 & \\
LII $\left({ }^{\circ}\right.$, SD $)$ & $187.4(8.6)$ & $183.0(6.9)$ & 0.001 \\
All $\left({ }^{\circ}\right.$, SD $)$ & $46.8(17.9)$ & $42.2(13.8)$ & 0.152 \\
\hline
\end{tabular}

* LII, lateral instability index

$\dagger$ All, anterior instability index

‡ OA, osteoarthritis

Table III. Comparison of $\mathrm{LII}^{*}$ and $\mathrm{AlI}^{\dagger}$ in pre-arthrosis and early-stage $\mathrm{OA}^{\ddagger}$ hips

\begin{tabular}{|c|c|c|c|}
\hline & Pre-arthrosis hips & Early OA hips & p-value \\
\hline Number of hips & 35 & 49 & \\
\hline LII $\left({ }^{\circ}, \mathrm{SD}\right)$ & $197.2(9.2)$ & $197.6(8.2)$ & 0.863 \\
\hline All $\left({ }^{\circ}, \mathrm{SD}\right)$ & $42.1(13.1)$ & $49.3(19.1)$ & 0.114 \\
\hline
\end{tabular}

* LII, lateral instability index

$\uparrow$ All, anterior instability index

† OA, osteoarthritis

either OA or unaffected hips. There was a significant difference between $\mathrm{OA}$ and unaffected hips with respect to the LII, but not the AII (Table II). In the OA group there was no difference between pre-arthrosis and early OA hips for either the AII or the LII (Table III). A high LII indicates lateral instability; hence these results show that the OA hips were more unstable laterally than the unaffected hips.

Factor analysis for progression of OA. Of the 22 women with unilateral pre-arthrosis, disease progression was seen in 12. Two hips progressed to early OA, six to advancedstage and four OA to terminal-stage required a total hip replacement (Table IV). The mean interval between the initial examination and a diagnosis of progression was 106 months (84 to 120 ).

Of the six factors, only LII was a significant independently variable factor associated with progression to OA (Table IV).

The threshold value of LII during progress to the early or more advanced stages of OA was calculated. Nagelkerke's $\mathrm{R}^{2}$ for LII (assuming LII as the single variable) was 0.50 . The estimated coefficient regression for LII against the
Table IV. Predictive value of six factors (performed using binary logistic analysis)

\begin{tabular}{lll}
\hline Factors $^{*}$ & OR $^{\dagger}\left(\mathbf{9 5 \%} \mathbf{C l}^{\ddagger}\right)$ & p-value \\
\hline Age & $4.467(0.892$ to 22.365$)$ & 0.0686 \\
LII & $1.229(1.031$ to 1.465$)$ & 0.0217 \\
All & $1.022(0.968$ to 1.079$)$ & 0.4315 \\
Sharp angle & $1.006(0.865$ to 1.169$)$ & 0.939 \\
Centre-edge angle & $0.832(0.643$ to 1.075$)$ & 0.1594 \\
AHI & $0.885(0.734$ to 1.067$)$ & 0.2012 \\
\hline * LII, lateral instability index; All, anterior instability \\
index; AHI, acetabular head index \\
† OR, odds ratio \\
\multicolumn{4}{l}{ ₹ Cl, confidence interval }
\end{tabular}

probability of progression was 0.206 , the standard error was 0.090 and probability 0.022 . The constant tears were 40.395, standard error 17.668 and probability 0.022 . Therefore, the probability of disease progression $(\mathrm{Y})$ is:

$$
\mathrm{Y}=1 / 1+\mathrm{e}^{-(40.395+0.206 X)}
$$

where $X$ equals the measured value of LII. If we assume that $\mathrm{Y}=0.50$, the corresponding value of $\mathrm{X}$, i.e. the threshold LII value that predicts disease progression, can be calculated as:

$$
X=40.395 / 0.206=196.09 \text {. }
$$

Therefore, if LII is greater than 196, disease progression is more likely to occur (Fig. 3).

\section{Discussion}

The aim of this study was to test two hypotheses: first, whether or not the relationship between the orientation of the acetabulum and the proximal femoral angles differed between dysplastic and normal hips, and second, if differences existed, how did they contribute to the progression of OA in the dysplastic hip.

In order to address the first question, we initiated a study to develop a method of measuring both the anterior and the lateral opening angles of the acetabulum using bidirectional plain radiographs. This method was shown to be reliable by comparing direct measurements from a cadaver pelvis with those from bidirectional radiographs of the same pelvis. ${ }^{23}$ 


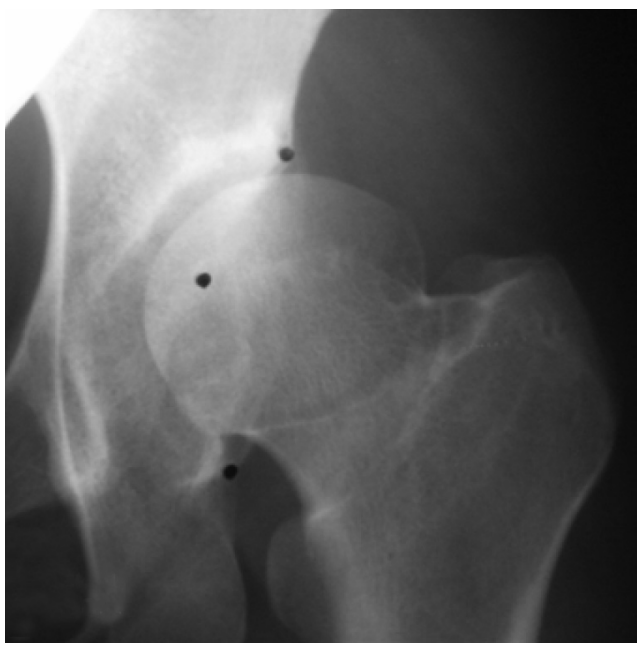

Fig. 3a

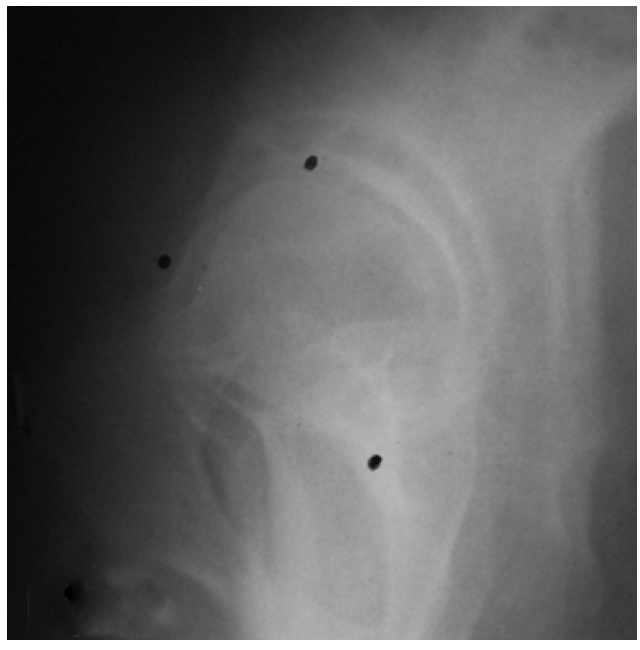

Fig. 3b

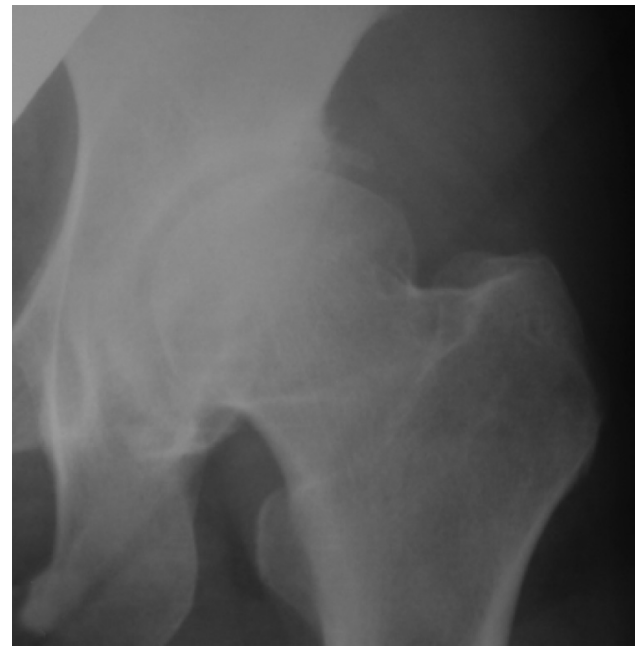

Fig. 3c

Radiographs showing the pre-arthrosis left hip of a 37-year-old woman (case 20). Figures 3a-posteroanterior (PA) radiograph showing an LII of 207. Figure 3b- lateral radiograph showing an All of 34. Figure 3c - PA radiograph at 120 months' followup showing progression of osteoarthritis to the advanced-stage.

We further applied this method in the clinical setting to evaluate the relationship between the acetabular inclination angles and the proximal femoral angles in cases of prearthrosis or early OA, and analysed the influence of these factors on the progression of OA changes. The reliability of this method was confirmed by high levels of inter- and intra-observer agreement.

We also measured femoral anteversion and the true femoral neck-shaft angles using bidirectional radiographs. In order to express this relationship quantitatively, we adopted the instability index proposed by $\mathrm{McKibbin}^{27}$ as a measure of instability of the dysplastic hip. He found in a study of the neonatal cadaver hip that reproduction of dislocation and reduction of the hip did not depend independently on the femoral anteversion angle or the anterior opening angle of the acetabulum, but on the sum of these angles, and proposed an instability index to express the poor containment of the hip. Reference has been made to this index for the evaluation of unstable static alignments between the acetabulum and the proximal femur by Tönnis and Heinecke ${ }^{30}$ and Fairbank et al. ${ }^{31}$ We defined this parameter as AII, and incorporated a new parameter, LII, defined as the sum of the lateral opening angle of the acetabulum and the femoral neck-shaft angle. Higher values of these two indices indicate a more tenuous containment of the femoral head in the acetabulum and hence greater instability, as shown in Figure 4.

In this study, we found no correlation between AII and LII in either the OA or the unaffected hips, thus confirming that these two indices are independent variables. There 


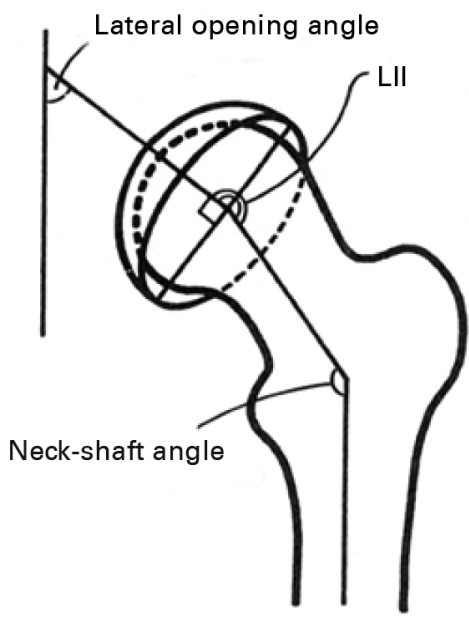

Fig. 4a

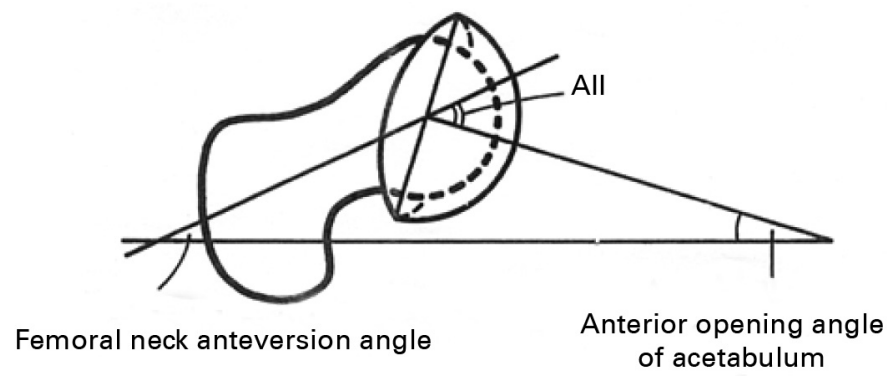

Fig. $4 b$

Diagrams illustrating the effect of larger lateral instability (LII) and anterior instability indices (AII). Figure 4a - a larger LII, defined as the sum of the lateral opening angle of the acetabulum and the neck-shaft angle of the femoral head, indicates decreased lateral containment of the hip and therefore increased lateral instability. Figure $4 \mathrm{~b}-\mathrm{a}$ larger All, defined as the sum of the anterior opening angle of the acetabulum and the anteversion angle of the femoral neck, indicates decreased anterior containment of the hip and therefore increased anterior instability.

was no significant difference in the AII between the OA and the unaffected hips. However, the LII was significantly higher in OA hips, indicating that lateral instability is more of a problem than anterior instability in such hips. With this significant difference in LII between OA and unaffected hips, we felt it reasonable to allow unaffected hips to be the control group, as radiological examination of normal adults solely to obtain a control series is not justified. Our findings suggest that the OA dysplastic hip has not been fully understood to date, given that previous evaluations of the relationship between acetabular orientation and the femoral neck angle have only measured the anterior opening angle of the acetabulum and the anteversion angle of the femoral neck. ${ }^{14-16}$

In order to address our second hypothesis, we examined how unstable alignment of the hip contributes to progression of the disease. No previous studies have reported on the value of the acetabular lateral opening and femoral neck angles in predicting the development of OA in the dysplastic hip.

From our prospective study of 22 patients with unilateral pre-arthrosis followed for a minimum of 15 years, we found that neither age at initial examination, centre-edge angle, acetabular head index, Sharp angle nor AII was associated with progression to OA. In contrast, LII was significantly associated with progression. This indicates that lateral instability is of greater concern than anterior instability in the dysplastic hip, and that lateral instability is involved in the progression of OA. Insufficient attention has been paid to the evaluation of lateral instability in previous studies.

Maeyama et $\mathrm{al}^{32}$ reported that the femoral head moves upwards, backwards and laterally during walking, and that a peri-acetabular osteotomy in the dysplastic hip reduces the magnitude of acceleration of the femoral head in the upward and lateral directions. Our study supports this theory, suggesting that a peri-acetabular osteotomy improves the lateral containment function of the acetabulum by reducing the LII. From these results we believe that the lack of lateral and superior stability, expressed by the LII in our study, would increase the magnitude of posterior and lateral acceleration, resulting in the progression of OA of the hip. We found that LII values higher than 196 predicted progression from preorthosis to full OA.

In this study, it was essential to take accurate bidirectional radiographs of the hips in order to measure the acetabular inclination angles correctly. The method described may only be used where there is not severe deformity of the femoral head or acetabulum, particularly of the acetabular rim, and in cases without contracture of the hip. Deformity at these sites indicates the onset of OA, therefore disease progression can be established without the need for the quantitative methods described here.

Our study shows that this method is easily applicable in clinical practice and gives a quantitative bi-directional morphological evaluation of the dysplastic hip. Accurate radiographs are needed, but the method is of use in predicting the progression from dysplasia to OA.

\section{Supplementary material}

$\because$ Two graphs showing the lateral instability index C plotted against the anterior instability index and a table detailing the clinical data of the 22 patients is available with the electronic version of this paper on our website at www.jbjs.org.uk 


\section{Listen live}

Listen to the abstract of this article at

www.jbjs.org.uk/interactive/audio

We thank Dr S. Tatsunami, Associate Professor of the Division of Medical Statistics at St Marianna University School of Medicine, for his kind support in analysis of the data.

No benefits in any form have been received or will be received from a com mercial party related directly or indirectly to the subject of this article.

\section{References}

1. Wiberg G. Studies on dysplastic acetabula and congenital subluxation of the hip joint: with special reference to the complication of osteoarthritis. Acta Chirurgica Scandinavica 1939;83(Suppl):7-135

2. Jacobsen S, Sonne-Holm S, Lund B, et al. Pelvic orientation and assessment of hip dysplasia in adults. Acta Orthop Scand 2004;75:721-9.

3. Heyman $\mathbf{C H}$, Herndon $\mathbf{C H}$. Legg-Perthes disease: a method for the measurement of the roentgenographic result. J Bone Joint Surg [Am] 1950;32-A:767-78.

4. Delaunay S, Dussault RG, Kaplan PA, Alford BA. Radiographic measurements of dysplastic adult hips. Skeletal Radiol 1997;26:75-81.

5. Li PL, Ganz R. Morphologic features of congenital acetabular dysplasia: one in six is retroverted. Clin Orthop 2003;416:245-53.

6. Mast JW, Brunner RL, Zebrack J. Recognizing acetabular version in the radiographic presentation of hip dysplasia. Clin Orthop 2004;418:48-53.

7. Hasegawa Y, Iwata H, Mizuno M, et al. The natural course of osteoarthritis of the hip due to subluxation or acetabular dysplasia. Arch Orthop Trauma Surg 1992;111:187-91.

8. Murphy SB, Ganz R, Müller ME. The prognosis in untreated dysplasia of the hip: a study of radiographic factors that predict the outcome. J Bone Joint Surg [Am]1995;77-A:985-9.

9. Nozawa M, Kurosawa H, Shitoto K, et al. Radiographic study of osteoarthritis of the hip joints treated conservatively for more than ten years. J Orthop Surg 1997;5:1-8.

10. Reijman M, Hazes JM, Pols HA, Koes BW, Bierman-Zeinstra SM. Acetabula dysplasia predicts incident osteoarthritis of the hip: the Rotterdam study. Arthritis Rheum 2005; $52: 787-93$

11. Cooperman DR, Wallensten R, Stulberg SD. Acetabular dysplasia in the adult. Clin Orthop 1983;175:79-85.

12. Hisatome T, Yasunaga $\mathbf{Y}$, Tanaka R, et al. Natural course of the minimally symptomatic nonoperated hip in patients with bilateral hip dysplasia treated with contralateral rotational acetabular osteotomy. J Orthop Sci 2005;10:574-80.

13. Nagasawa F, Miyake Y, Akazawa $\mathbf{H}$, et al. Predictability of the progress of secondary osteoarthritis after developmental dislocation of the hip, utilizing inferior edge (of the teardrop) center (of the femoral head) distance. J Orthop Sci 2000;5:10-17.

14. Reikerås $\mathbf{0}$, Bjerkreim I, Kolbenstvedt A. Anteversion of the acetabulum and femoral neck in normals and in patients with osteoarthritis of the hip. Acta Orthop Scand 1983;54:18-23
15. Anda S, Terjesen T, Kvistad KA. Computed tomography measurements of the acetabulum in adult dysplastic hips: which level is appropriate? Skeletal Radiol 1991;20:267-71.

16. Anda S, Teriesen T, Kvistad KA, Svenningsen S. Acetabular angles and femoral anteversion in dysplastic hips in adults: CT investigation. J Comput Assist Tomogr 1991;15:115-20.

17. Johnston CE 2nd, Wenger DR, Roberts JM, Burke SW, Roach JW. Acetabular coverage: three-dimensional anatomy and radiographic evaluation. J Pediatr Orthop 1986;6:548-58.

18. Azuma $\mathbf{H}$, Taneda $\mathbf{H}$, Igarashi $\mathbf{H}$. Evaluation of acetabular coverage: three-dimensional CT imaging and modified pelvic inlet view. J Pediatr Orthop 1991;11:765-9.

19. Murphy SB, Kijewski PK, Millis MB, Harless A. Acetabular dysplasia in the adolescent and young adult. Clin Orthop 1990;261:214-23.

20. Abel MF, Sutherland DH, Wenger DR, Mubarak SJ. Evaluation of CT scans and 3-D reformatted images for quantitative assessment of the hip. J Pediatr Orthop 1994; 14:48-53.

21. Dandachli W, Kannan V, Richards R, et al. Analysis of cover of the femoral head in normal and dysplastic hips: new CT-based technique. J Bone Joint Surg [Br] 2008;90-B:1428-34.

22. Argenson JN, Flecher $\mathbf{X}$, Parratte S, Aubaniac JM. Anatomy of the dysplastic hip and consequences for total hip arthroplasty. Clin Orthop 2007;465:50-5.

23. Nagao Y, Aoki H, Ishii SJ, Masuda T, Beppu M. Radiographic method to measure the inclination angle of the acetabulum. J Orthop Sci 2008;13:62-71.

24. Kai M. Roentgenographic measurement of proximal end of the femur and its clinical application. Jpn J Orthop Surg 1937;12:389-497 (in Japanese).

25. Ogata K, Goldsand EM. A simple biplanar method of measuring femoral anteversion and neck-shaft angle. J Bone Joint Surg [Am] 1979;61-A:846-51.

26. Takatori $Y$, Ito $K$, Sofue $M$, et al; Investigation Group into Coxarthrosis and Acetabular Dysplasia in Japan. Analysis of interobserver reliability for radiographic staging of coxarthrosis and indexes of acetabular dysplasia: a preliminary study. J Orthop Sci 2010;15:14-19

27. McKibbin B. Anatomical factors in the stability of the hip joint in the newborn. $J$ Bone Joint Surg [Br] 1970;52-B:148-59

28. Sharp IK. Acetabular dysplasia: the acetabular angle. J Bone Joint Surg [Br]1961;43B:268-72.

29. Nagelkerke NJD. A note on a general definition of the coefficient of determination. Biometrika 1991;78:691-2.

30. Tönnis D, Heinecke A. Acetabular and femoral anteversion: relationship with osteoarthritis of the hip. J Bone Joint Surg [Am]1999;81-A:1747-70.

31. Fairbank JC, Howell P, Nockler I, Lloyd-Roberts GC. Relationship of pain to the radiological anatomy of the hip joint in adults treated for congenital dislocation of the hip as infants: a long-term follow-up of patients treated by three methods. J Pediatr Orthop 1986;6:539-47.

32. Maeyama A, Naito M, Moriyama S, Yoshimura I. Periacetabular osteotomy reduces the dynamic instability of dysplastic hips. J Bone Joint Surg [Br] 2009;91B:1438-42. 